\title{
Multi-period Investment Strategies with Transaction Costs Under Cumulative Prospect Theory
}

\author{
Liurui Deng ${ }^{1}$, Lan Yang ${ }^{1} \&$ Bolin $\mathrm{Ma}^{2}$ \\ ${ }^{1}$ Business School, Hunan Normal University, China \\ ${ }^{2}$ College of Mathematics, Physics and Information Engineering, Jiaxing University, China \\ Correspondence: Liurui Deng, Business School, Hunan Normal University, China. E-mail: purplerosed@yahoo.com
}

Received: May 30, 2019

doi:10.11114/afa.v5i2.4432

\author{
Accepted: August 6, $2019 \quad$ Available online: August 15, 2019 \\ URL: https://doi.org/10.11114/afa.v5i2.4432
}

\begin{abstract}
This paper focuses on optimal investment strategies under cumulative prospect theory (CPT). Considering transaction costs, we investigate CPT investors multi-period optimal portfolios. Our main contributions relative to previous work are expanding a single-period optimization problem to a multi-period optimization problem and investigating the impact of transaction costs on optimal portfolio selections. In a numerical analysis that applied original data on four stocks from the NASDAQ, we examine the effects of different risks on the optimal portfolio. Moreover, in contrast with the results without transaction costs, we come to conclusion that the optimal strategy with transaction costs is less sensitive to risk.
\end{abstract}

Keywords: Cumulative prospect theory (CPT), multi-period investment strategy, CPT investor, portfolio selection, transaction cost, probability weighting function

\section{Introduction}

In traditional research, investment strategies rely on expected utility theory (EUT). The basic assumption of EUT is that investors are rational and that probability is linear, regardless of subjective probability (see Neummann and Morgenstern, 1944). In fact, we find that the risk appetite behavior of many investors are irrational and inconsistent, which can be verified by empirical research. However,EUT assumes the opposite. Investors decisions are often impacted by their emotion, experience, behavior habits, thinking way, and so on. We notice that CPT (see Tversky and Kahneman, 1992b)more accurately models the characteristics of the investors psychology and behavior. Specially, there are three differences from the traditional theory. The first one is that the investors attitudes for risk change rather than keep risk aversion. Usually, the investors exhibit risk aversion on gains and exhibit risk seeking on losses. The second one is that the investorss valuations of risk assets are related to a reference point, not considering absolute wealth level in classical theories. The last one is that the investors dislike losses, so people are more sensitive to loss than to gain. To investigate the optimal investment strategy which is closer to investors psychology and behavior, we will research the investment strategy under the CPT framework. Under CPT framework, some researchers discuss the similar investment strategies to ours.By building a mathematical model, Jin and Zhou obtained the optimal portfolio in continuous time under the CPT background(see Jin and Zhou, 2008). Based on Jins research, Bernard et al. observed the sensitivity of CPT investors to risk excess returns in discrete time and their optimal portfolio (see Bernard and Ghossoub, 2009). Unlike other literature, we developed an approach which can define loss aversion and analyze portfolio sensitivity to brand new reference points (see He and Zhou, 2011a,b). Pirvu and Schulze applied the definition of the measure to the case of multi-asset (see Pirvu and Schulze, 2012). All of these previous work focus on the optimal portfolio in a single-period. However, in financial market, from an initial time to a terminal time, investors often have much chances

to change portfolio selections to maximize their returns at the terminal time. So, we are interested in the optimal strategy in multiple periods and formulating dynamic strategy to characterize the change of portfolio selections. To the best of our knowledge, Carassus and Rsonyi study on multi-period portfolio decisions in discrete time, which further investigated Jin's work. And they offered possible conditions for the conclusion to be verified (see Carassus and Rasonyi, 2013). Shi et al. extended the discrete model to a multi-period condition and solved for the first time the problem of time inconsistency caused by the complexity of the model (see Shi et al., 2014). For a time-changing benchmark, Deng and Pirvu took into account the manager's limits on the number of trades (see Deng and Pirvu, 2015). 
Observing these previous works, we find that these do not discuss transaction costs. However, a real market is frictional. That is, transaction costs really exist in reality. More- over, without transaction costs, as for Mertons research, the optimal portfolio may lead to unrealistic strategies such as investing infinite amount in risky assets. Hence, we hope to explore the CPT strategy with transaction costs. Although some researchers have investigated the optimal portfolio with transaction costs under the EUT framework, (see Davis \& Norman, 1990; Kabanov \& Safarian, 2010; Magill \& Constantinides, 1976; Shreve \& Soner, 1994), few researchers have been solved this puzzle under the CPT framework. Zou and Zagst first proposes single-period optimal investment with transaction costs under the CPT framework (see Zou \& Zagst, 2017) Our work expands their result to a multi-period problem.

Our research has to face the enormous challenge that is how to choose a suitable bench- mark. Compared with the optimal strategy in a single period, our benchmark is a time- changing benchmark. Specially, in Zou and Zagst's research, investors only need to make one decision at an initial time. So, when choosing a benchmark, they do not need to consider the impact of the time on the benchmark. But, in our research, from an initial time to a terminal time, investors may make many different decisions at the different time. Therefore, when choosing a benchmark, we should consider the effect of the different time points on the benchmark and choose a time-changing benchmark to accurately characterize the psychological changes of investors at different time points. Furthermore, we propose the time inconsistency of optimal portfolio selection based on the time-changing benchmark. Compared with the optimal strategy without transaction costs under CPT, when choosing the benchmark, we should consider the effects of trade cost on the benchmark. Because the amounts of the investments at the different time points are different, the amount of the transaction costs at the different time points are different too. Therefore, when choosing the benchmark, we consider not only the time-changing wealth level but also the time-changing transaction costs. In numerical analysis, we choose four stocks from the NASDAQ which have the different levels of risks and the different levels of risk volatilities, we examine the sensitivity of the optimal portfolios for the risks of the risky assets. Moreover, we compare this with a no-transaction strategy. We find that the strategy without transaction costs is more sensitive for risks than one with transaction costs regardless of different levels of risks and risk volatilities. The result is consistent with the classical conclusion that transaction costs reduce the liquidity of assets.

Through detailed comparisons to the previous literatures, we summarize our main contributions as follows.

- We research the multi-period optimal strategy under a frictional market. As mentioned earlier, except for Zou and Zagst, few researchers propose the CPT-investors' strategies under frictional markets. However, without transaction costs in frictional markets, the optimal strategy will lead to some unrealized results, such as investing infinite amount of money in risky assets. Thus, when we discuss the optimal portfolios under CPT, it is necessary to consider transaction costs. Although Zou and Zagst also discuss consider transaction costs, their result is only suitable for a single period. Our result is applicable not only to the single period but also to multiply periods.

- We propose a different benchmark from previous works. Compared with Zou and Zagst's benchmark, our benchmark is time-changing. That is, our benchmark is related to the change of decision time. Because Zou and Zagst discuss the optimal problem under single period and an investor makes a decision at an initial time, they do not need to consider the impact of time on benchmark. But we investigate the optimal portfolio under the multi-period, we have to face the time-changing benchmark. Compared with other previous CPT strategies except for Zou and Zagsts research, we discuss the effects of trade cost on the benchmark. Moreover, transaction costs are also time-changing. Because previous works only consider frictionless market, transaction costs do not need to be considered. However, trade costs are presented in the real market. And, without trade costs, the strategies will lead to unreal results. So it is necessary to consider the transaction cost in a frictional market.

- We investigate the time inconsistency of the strategy. Due to the time-changing benchmark, we have to face the time inconsistency of the strategy. Using the backward induction, we get the recursive solution of the optimal portfolio. Compared with previous research, our result reflects the influence of transaction costs on the strategy. Furthermore, numerical analysis shows that the optimal strategy with transaction costs is less sensitive for risks than without transaction costs. This result shows that trade costs can reduce the liquidity of risky assets.

The rest of this paper is organized as follows: In Section 2, we present the basic setup. Section 3 provides the problems. The solutions are presented in Section 4. Numerical analysis is showed in Section 5. Section 6 draws the conclusion. The proofs are attached in the appendix.

\section{The Basic Setup}

We think about a financial market in which one can invest in one risk-free asset and one risky asset. The range of investment periods is $[0, T]$, where $T$ is a finite deterministic positive constant. We set a variable to represent investment time, called $t$, which is donated by discrete values $(t=0,1, \ldots, T-1)$. Trading the risk-free asset is frictionless, but 
investors trading in risky assets result in a fixed percentage of trade costs. Let's call that $\lambda$. Moreover, let $r_{t}$ denote the yield on the risk-free asset from time $t$ to time $t+1 . x_{t}$ and $y_{t}$ are respectively the initial capital of the risk-free asset and the risk asset at time $t$. The investor additionally invests the quantity of money, $\theta_{t}$, in the risk asset at time $t$. We denote the investor's wealth at time $t+1$ after liquidation by $W_{t+1}$. A straightforward computation leads to

$$
W_{t+1}=\left(1+r_{t}\right)\left(x_{t}-\theta_{t}\right)+\left(1+R_{t}\right)\left(y_{t}+\theta_{t}\right)-\lambda\left[\left(1+R_{t}\right)\left(y_{t}+\theta_{t}\right)^{+}+\left(1+r_{t}\right) \theta_{t}^{-}\right]
$$

Here, $R_{t}$ is a random variable that represents the yield on the risky asset from time $t$ to time $t+1$. The return process $\left\{R_{t}\right\}_{\{t=0,1, \ldots, T-1\}}$ is an adapted stochastic process defined over the probability space $\left(\Omega, \mathcal{F}_{t}, \mathcal{F}, P\right)$. The information set from period $t$ is $\mathcal{F}_{t}=\sigma\left(R_{0}, R_{1}, \ldots, R_{t-1}\right)$. Moreover, $R_{t} \in \mathcal{F}_{t+1}$.

\subsection{The Benchmark Wealth}

If the initial time is $t$ and the terminal time is $k$, we let the benchmark be

$$
B_{t}^{k}=\left(1+r_{t}\right)\left(1+r_{t+1}\right) \ldots\left(1+r_{k-1}\right) x_{t}+\left(1+R_{t}\right)\left(1+R_{t+1}\right) \ldots\left(1+R_{k-1}\right)\left(y_{t}-\lambda y_{t}^{+}\right) .
$$

Remark 0.1. It is easy to find that this benchmark is related to the initial time. We choose this benchmark in order to accurately characterize the psychological changes of investors at the different initial times.

Remark 0.2. If $t=k-1$, then $B_{k-1}^{k}=\left(1+r_{k-1}\right) x_{k-1}+\left(1+R_{k-1}\right)\left(y_{k-1}-\lambda y_{k-1}^{+}\right)$. This result is consistent with Zou and Zagst's $B$ (see Zou \& Zagst, 2017).

Let

$$
\begin{aligned}
z_{t}^{k} & =(1-\lambda)\left(1+R_{t}\right)\left(1+R_{t+1}\right) \ldots\left(1+R_{k-1}\right)-\left(1+r_{t}\right)\left(1+r_{t+1}\right) \ldots\left(1+r_{k-1}\right) I_{\theta_{t} \geq 0} \\
& +(1-\lambda)\left(R_{t}-r_{t}\right)\left(R_{t+1}-r_{t+1}\right) \ldots\left(R_{k-1}-r_{k-1}\right) I_{\theta_{t}<0},
\end{aligned}
$$

where $I$ is an indicative function of $\theta_{t}$.

Remark 0.3. If $t=k-1$, then

$$
z_{k-1}^{k}=(1-\lambda)\left(1+R_{k-1}\right)-\left(1+r_{k-1}\right) I_{\theta_{t} \geq 0}+(1-\lambda)\left(R_{k-1}-r_{k-1}\right) I_{\theta_{t}<0} .
$$

This result is consistent with Zou and Zagst's $Z_{1}$ and $Z_{2}$ (see Zou \& Zagst, 2017). A small difference is that we write $Z_{1}$ and $Z_{2}$ as a unified form, $z_{k-1}$, for later simplicity.

The benchmark wealth at time $t+1$, given the initial time $t$, is

$$
\bar{W}_{t}^{t+1}=W_{t+1}-B_{t}^{t+1}=z_{t}^{t+1} \theta_{t}
$$

This result is consistent with Zou and Zagst's result (see Zou \& Zagst, 2017).

In other words, our benchmark and benchmark wealth are consistent with Zou and Zagst's result in a single period (see Zou \& Zagst, 2017).

Given the initial time $t$, the benchmark wealth at time $t+2$ is

$$
\begin{aligned}
\bar{W}_{t}^{t+2} & =W_{t+2}-B_{t}^{t+2} \\
& =\left(1+r_{t+1}\right)\left(x_{t+1}-\theta_{t+1}\right)+\left(1+R_{t+1}\right)\left(y_{t+1}+\theta_{t+1}\right) \\
& -\lambda\left[\left(1+R_{t+1}\right)\left(y_{t+1}+\theta_{t+1}\right)^{+}+\left(1+r_{t+1}\right) \theta_{t+1}^{-}\right] \\
& -\left(1+r_{t}\right)\left(1+r_{t+1}\right) x_{t}-\left(1+R_{t}\right)\left(1+R_{t+1}\right)\left(y_{t}-\lambda y_{t}^{+}\right) .
\end{aligned}
$$

Because

$$
x_{t+1}=\left(1+r_{t}\right)\left(x_{t}-\theta_{t}\right)
$$

and

$$
y_{t+1}=\left(1+R_{t}\right)\left(y_{t}+\theta_{t}\right),
$$

we have

$$
\begin{aligned}
\bar{W}_{t}^{t+2} & =(1-\lambda)\left(1+R_{t}\right)\left(1+R_{t+1}\right) \theta_{t}-\left(1+r_{t}\right)\left(1+r_{t+1}\right) \theta_{t} \\
& +(1-\lambda)\left(1+R_{t+1}\right) \theta_{t+1}-\left(1+r_{t+1}\right) \theta_{t+1} \\
& =z_{t}^{t+2} \theta_{t}+z_{t+1}^{t+2} \theta_{t+1}
\end{aligned}
$$

In a general way, the benchmark wealth is determined by the following Proposition. 
Proposition 0.1. If the initial time is $t(t=0,1,2, \ldots, T-1)$, then the benchmark wealth at $T$ is

$$
\bar{W}_{t}^{T}=z_{t}^{T} \theta_{t}+z_{t+1}^{T} \theta_{t+1}+\ldots+z_{T-1}^{T} \theta_{T-1}
$$

Proof. The proof is annexed to the appendix A.

This kind of benchmarking leads to time-inconsistent behavior, as outlined in Remark 0.4. The resolution we propose is to consider benchmarking at time $T-1$ only $^{1}$. Thus, if the initial time is $t(t=0,1,2, \ldots, T-1)$, then the benchmark wealth at $T$ is

$$
\underline{W}_{t}^{T}=z_{T-1}^{T} \theta_{T-1} \quad \text { assume the initial time is } t .
$$

This benchmark wealth is important for expanding a single-period problem to a multi-period problem. Based on this process, we attain a recursion formula of the optimization solution in multiple periods.

\subsection{The CPT Risk Criterion}

Investors get positive utility for gains and negative utility for losses. The benchmark differentiates gains and losses. The formal definition is as follows.

Definition 0.1. (see (Tversky \& Kahneman, 1992a; Tversky \& Kahneman, 1992b)) The value function $u$ is defined as:

$$
u(x)= \begin{cases}u^{+}(x) & \text { if } x \geq 0, \\ -u^{-}(-x) & \text { if } x<0\end{cases}
$$

where $u^{+}: \overline{\mathbb{R}}^{+} \rightarrow \overline{\mathbb{R}}^{+}$and $u^{-}: \overline{\mathbb{R}}^{+} \rightarrow \overline{\mathbb{R}}^{+}$satisfy:

(i) $u(0)=u^{+}(0)=u^{-}(0)=0$;

(ii) $u^{+}(+\infty)=u^{-}(+\infty)=+\infty$;

(iii) $u^{+}(x)=x^{\alpha}$, with $0<\alpha<1$ and $x \geq 0$;

(iv) $u^{-}(x)=\beta x^{\alpha}$ with $\beta>1$, and $x \geq 0$.

Definition 0.2. Let $F_{X}(\cdot)$ be the cumulative distribution function (CDF) of a random variable $X$. We define the two probability weighting functions $T^{+}:[0,1] \rightarrow[0,1]$ and $T^{-}:[0,1] \rightarrow[0,1]$ as follows:

$$
\begin{aligned}
& T^{+}\left(F_{X}(x)\right)=\frac{F_{X}^{\gamma}(x)}{\left(F_{X}^{\gamma}(x)+\left(1-F_{X}(x)\right)^{\gamma}\right)^{1 / \gamma}}, \text { with } 0.28<\gamma<1, \\
& T^{-}\left(F_{X}(x)\right)=\frac{F_{X}^{\delta}(x)}{\left(F_{X}^{\delta}(x)+\left(1-F_{X}(x)\right)^{\delta}\right)^{1 / \delta}}, \text { with } 0.28<\delta<1 .
\end{aligned}
$$

Definition 0.3. The objective function of CPT investors is expressed by $U(W)$, as

$$
U(W)=\int_{0}^{+\infty} T^{+}\left(1-F_{W}(x)\right) d u^{+}(x)-\int_{0}^{+\infty} T^{-}\left(F_{W}(-x)\right) d u^{-}(x),
$$

where $W$ is the benchmark wealth. $U(W)$ is a sum of two Choquet integrals (see Choquet (1953) and Chateauneuf et al. (2000)).

It is well-defined when

$$
\alpha<2 \min (\delta, \gamma)
$$

From Definition 0.3, it follows that the objective function of the CPT investor at time $t$ is

$$
U\left(\bar{W}_{t}^{T}\right)=\int_{0}^{+\infty} T^{+}\left(1-F_{\bar{W}_{t}^{T}}(x)\right) d u^{+}(x)-\int_{0}^{+\infty} T^{-}\left(F_{\bar{W}_{t}^{T}}(-x)\right) d u^{-}(x) .
$$

\footnotetext{
${ }^{1}$ This assumption is intended to make the portfolio problem easy to handle.
} 
As we show in Remark 0.4, this risk criterion is time-inconsistent; therefore, we recommend that the benchmark wealth $\bar{W}_{t}^{T}$ and the objective function

$$
U\left(\underline{W}_{t}^{T}\right)=\int_{0}^{+\infty} T^{+}\left(1-F_{\underline{W}_{t}^{T}}(x)\right) d u^{+}(x)-\int_{0}^{+\infty} T^{-}\left(F_{\underline{W}_{t}^{T}}(-x)\right) d u^{-}(x) .
$$

\section{The Problems}

In this section, we develop objective for CPT-investor. In the first step, let's start with the one period model.

\subsection{The Single-period Problem}

We assume that the current time is $T-1$. Think back to the benchmark wealth at time $T$ is

$$
\bar{W}_{T-1}^{T}=z_{T-1}^{T} \theta_{T-1}=\underline{W}_{T-1}^{T} .
$$

The objective of CPT investors is to address the following portfolio issues

$$
\begin{aligned}
& (P) \quad \max _{\theta_{T-1} \in \overline{\mathcal{F}}_{T-1}} U\left(\bar{W}_{T-1}^{T}\left(\theta_{T-1}\right)\right) \\
& =\max _{\theta_{T-1} \in \overline{\mathcal{F}}_{T-1}}\left[\int_{0}^{+\infty} T^{+}\left(1-F_{\bar{W}_{T-1}^{T}}(x)\right) d u^{+}(x)-\int_{0}^{+\infty} T^{-}\left(F_{\bar{W}_{T-1}^{T}}(-x)\right) d u^{-}(x)\right],
\end{aligned}
$$

and to find the optimal portfolio $\theta_{T-1}^{*}$

$$
\begin{aligned}
\theta_{T-1}^{*} & =\arg \max _{\theta_{T-1} \in \overline{\mathcal{F}}_{T-1}} U\left(\bar{W}_{T-1}^{T}\left(\theta_{T-1}\right)\right) \\
& =\arg \max _{\theta_{T-1} \in \overline{\mathcal{F}}_{T-1}}\left[\int_{0}^{+\infty} T^{+}\left(1-F_{\bar{W}_{T-1}^{T}}^{T}(x)\right) d u^{+}(x)-\int_{0}^{+\infty} T^{-}\left(F_{\bar{W}_{T-1}^{T}}^{T}(-x)\right) d u^{-}(x)\right] .
\end{aligned}
$$

\subsection{The Multi-period Problem}

In order to obtain time-consistent optimal strategies, we work with the benchmark wealth $\underline{W}_{t}^{T}$. The objective of CPT investors is to address the following portfolio issues

$$
\begin{aligned}
& \max _{\theta_{i} \in \overline{\mathcal{F}}_{i}, i=t, t+1, \ldots T-1} U\left(\underline{W}_{t}^{T}\left(\theta_{i}\right)\right) \\
& =\max _{\theta_{i} \in \overline{\mathcal{F}}_{i}, i=t, t+1, \ldots T-1}\left[\int_{0}^{+\infty} T^{+}\left(1-F_{\underline{W}_{t}^{T}}(x)\right) d u^{+}(x)-\int_{0}^{+\infty} T^{-}\left(F_{\underline{W}_{t}^{T}}(-x)\right) d u^{-}(x)\right] .
\end{aligned}
$$

Remark 0.4. If the benchmark wealth $\bar{W}_{t}^{T}$ is used, then the optimal strategy is time-inconsistent. That is, unless there is a commitment mechanism, the optimal strategy calculated in the past is not implemented. Indeed, $\theta_{T-1}^{*} \neq \hat{\theta}_{T-1}$, where

$$
\theta_{T-1}^{*}=\arg \max _{\theta_{T-1} \in \overline{\mathcal{F}}_{T-1}} U\left(\bar{W}_{T-1}^{T}\left(\theta_{T-1}\right)\right)
$$

and

$$
\left(\hat{\theta}_{T-2}, \hat{\theta}_{T-1}\right)=\arg \max _{\theta_{T-2}, \theta_{T-1} \in \overline{\mathcal{F}}_{T-2}} U\left(\bar{W}_{T-2}^{T}\left(\theta_{T-2}, \theta_{T-1}\right)\right) .
$$

\section{The Solutions}

Our presentation begins with the single-period case.

\subsection{The Single-period Solution}

First, we discuss the optimal portfolio at initial time $T-1$. 
Proposition 0.2. The portfolio optimization problem is transformed into

$$
\max _{\theta_{T-1} \in \overline{\mathcal{F}}_{T-1}, \theta_{T-1} \geq 0} U\left(\bar{W}_{T-1}^{T}\left(\theta_{T-1}\right)\right)=W_{T-1}^{\alpha} A_{T-1} I_{W_{T-1} \geq 0}-\left(-W_{T-1}\right)^{\alpha} \alpha B_{T-1} I_{W_{T-1}<0},
$$

where

$$
\begin{gathered}
A_{T-1}=\max _{z \in(-\infty,+\infty)} g_{T-1}(s), \\
B_{T-1}=-\max _{z \in(-\infty,+\infty)} l_{T-1}(s), \\
g_{T-1}(s)= \begin{cases}s^{\alpha} h(T-1) I_{s \in[0,+\infty)} & \text { if } \theta_{T-1} \geq 0, \\
(-s)^{\alpha} k(T-1) I_{s \in(-\infty, 0]} & \text { if } \theta_{T-1}<0,\end{cases} \\
l_{T-1}(s)= \begin{cases}(-s)^{\alpha} h(T-1) I_{s \in(-\infty, 0]} & \text { if } \theta_{T-1} \geq 0, \\
(-s)^{\alpha} k(T-1) I_{s \in(-\infty, 0]} & \text { if } \theta_{T-1}<0,\end{cases} \\
h(T-1)=\int_{0}^{+\infty} T^{+}\left(1-F_{z_{T-1}^{T}}(x)\right) d u^{+}(x)-\int_{0}^{+\infty} T^{-}\left(F_{z_{T-1}^{T}}(-x)\right) d u^{-}(x)
\end{gathered}
$$

and

$$
k(T-1)=\int_{0}^{+\infty} T^{+}\left(1-F_{-z_{T-1}^{T}}(x)\right) d u^{+}(x)-\int_{0}^{+\infty} T^{-}\left(F_{z_{T-1}^{T}}(x)\right) d u^{-}(x) .
$$

Proof. The proof is annexed to the appendix B.

Theorem 0.3. When $\theta_{T-1} \geq 0$, the optimal CPT-investment strategy is

$$
\theta_{T-1}^{*}= \begin{cases}k_{T-1}^{*} W_{T-1} & \text { if } W_{T-1} \geq 0 \\ \hat{k}_{T-1}^{*} W_{T-1} & \text { if } W_{T-1}<0\end{cases}
$$

where

$$
k_{T-1}^{*}=\arg \max _{s \in(-\infty,+\infty)} g_{T-1}(s), \quad \hat{k}_{T-1}^{*}=\arg \max _{s \in(-\infty,+\infty)} l_{T-1}(s)
$$

Proof. The above conclusion can be obtained from proposition 0.2.

Remark 0.5. Here, our single-period solution takes a different form from Zou and Zagst' s solution (see Zou \& Zagst, 2017). In fact, our solution is consistent with theirs in essence. We propose this solution to derive the multi-period solution from this single-period solution.

\subsection{The Multi-period Solution}

We can get the following key Proposition and Theorem for multiple periods.

Proposition 0.4. Given initial time t, the optimal CPT value is obtained through recursion

$$
\max _{\theta_{t}, \theta_{t+1}, \ldots \theta_{T-1}} U\left(\underline{W}_{t}^{T}\right)=A_{t} W_{t}^{\alpha} I_{W_{t} \geq 0}-B_{t}\left(-W_{t}\right)^{\alpha} I_{W_{t}<0}
$$

where

$$
\begin{gathered}
A_{t}=\max _{s \in(-\infty,+\infty)} g_{t}(s), \\
B_{t}=-\max _{s \in(-\infty,+\infty)} l_{t}(s),
\end{gathered}
$$




$$
\begin{aligned}
g_{t}(s)= & E\left[\left(\left(1+r_{t}\right)(1-s)+\left(1+R_{t}\right) s-\lambda\left(1+R_{t}\right) s^{+}\right.\right. \\
+ & \left.\lambda\left(1+r_{t}\right)\left(s-y_{t}\right) I_{\theta_{t}<0}\right)^{\alpha} A_{t+1} \\
& I_{\left(1+r_{t}\right)(1-s)+\left(1+R_{t}\right) s-\lambda\left(1+R_{t}\right) s^{+}+\lambda\left(1+r_{t}\right)\left(s-y_{t}\right) I_{\theta_{t}<0} \geq 0} \\
- & \left(-\left(1+r_{t}\right)(1-s)-\left(1+R_{t}\right) s+\lambda\left(1+R_{t}\right) s^{+}\right. \\
- & \left.\lambda\left(1+r_{t}\right)\left(s-y_{t}\right) I_{\theta_{t}<0}\right)^{\alpha} B_{t+1} \cdot \\
& \left.I_{\left(1+r_{t}\right)(1-s)+\left(1+R_{t}\right) s-\lambda\left(1+R_{t}\right) s^{+}+\lambda\left(1+r_{t}\right)\left(s-y_{t}\right) I_{\theta_{t}<0}<0} \mid \mathcal{F}_{t}\right]
\end{aligned}
$$

and

$$
\begin{aligned}
l_{t}(s)= & E\left[\left(\left(1+r_{t}\right)(s-1)+\left(1+R_{t}\right)(-s)-\lambda\left(1+R_{t}\right)(-s)^{+}\right.\right. \\
+ & \left.\lambda\left(1+r_{t}\right)\left(y_{t}-s\right) I_{\theta_{t}<0}\right)^{\alpha} A_{t+1} . \\
& I_{\left(1+r_{t}\right)(s-1)+\left(1+R_{t}\right)(-s)-\lambda\left(1+R_{t}\right)(-s)^{+}+\lambda\left(1+r_{t}\right)\left(y_{t}-s\right) I_{\theta_{t}<0}>0} \\
- & \left(-\left(1+r_{t}\right)(s-1)-\left(1+R_{t}\right)(-s)+\lambda\left(1+R_{t}\right)(-s)^{+}\right. \\
- & \left.\lambda\left(1+r_{t}\right)\left(y_{t}-s\right) I_{\theta_{t}<0}\right)^{\alpha} B_{t+1} . \\
& \left.I_{\left(1+r_{t}\right)(s-1)+\left(1+R_{t}\right)(-s)-\lambda\left(1+R_{t}\right)(-s)^{+}+\lambda\left(1+r_{t}\right)\left(y_{t}-s\right) I_{\theta_{t}<0} \leq 0} \mid \mathcal{F}_{T-2}\right] .
\end{aligned}
$$

Proof. The proof is annexed to the appendix C.

The following Theorem is the main result of the paper.

Theorem 0.5 . The optimal CPT-investment strategy $\left(\theta_{0}^{*}, \theta_{1}^{*}, \ldots, \theta_{T}^{*}\right)$ is given recursively for $t=T-1, T-2, \ldots, 0$ by

$$
\begin{gathered}
\theta_{t}^{*}= \begin{cases}k_{t}^{*} W_{t}-y_{t} & \text { if } W_{t} \geq 0, \\
\hat{k}_{t}^{*} W_{t}-y_{t} & \text { if } W_{t}<0,\end{cases} \\
k_{t}^{*}=\arg \max _{s \in(-\infty,-\infty)} g_{t}(s)
\end{gathered}
$$

and

$$
\hat{k}_{t}^{*}=\arg \max _{z \in(-\infty,-\infty)} l_{t}(s) \text {. }
$$

Proof. The above conclusion can be induced from the proof of Proposition 0.4.

Remark 0.6. The optimal solution is related to both the initial level of wealth and the initial monetary amount of risk assets. This solution gives us the following two key conclusions.

i) When $\theta_{t}^{*} \geq 0$, the increased initial monetary amount of risk assets is proportional to the initial level of wealth. That is, as initial wealth increases, so does investment in risky assets. Additionally, as the initial amount of money in the risky asset decreases, investment in the risky asset increases because under the circumstances, the optimal amount of money in the risky asset is more than the initial amount of money in the risk asset. Thus, the amount of money added to the risky asset is equal to the optimal amount of money in the risky asset minus the initial amount of money in the risky asset.Less initial money in the risk asset leads to a greater increase in money in the risk asset.

ii) When $\theta_{t}^{*}<0$, the money in the risky asset decreases and the money in the risk-free asset increases. The increased amount of money in the risk-free asset is proportional to the initial level of wealth. That is, as initial wealth increases, the increase in the investment in the risk-free asset increases. Additionally, as the initial money in the risky asset increases, the increase in the investment in the risk-free asset increases. In this case, the optimal amount of money in the risky asset is less than the initial amount of money in the risky asset, so the increased amount of money in the risk-free asset equals the initial amount of money in the risky asset minus the optimal amount of money in the risky asset. Therefore, the greater initial money in the risky asset leads to a increase in money in the risk-free asset.

\section{Numerical Analysis}

In this section, using empirical data and statistical tests, we compare our results with the results without transaction costs (Please see Figure 1). We set $\gamma=0.61, \delta=0.69, \lambda=0.003, \alpha=0.88$ and $\beta=2.2$. We choose the original data on four 
stocks from NASDAQ from February 1, 2017, to April 30, 2017: Autodesk (ADSK), Alexion Pharmaceuticals (ALXN), Applied Materials (AMAT), and Bed Bath and Beyond (BBBY).

In Figure 1, applying our results in a multi-period financial market, we obtain the optimal portfolios with transaction costs. Besides, using Deng and Pirvu's results (see Deng \& Pirvu, 2015) in a multi-period financial market, we obtain the optimal portfolios without transaction costs. Based on these results, we analyze the similarities and the differences between them and explain why they occur.
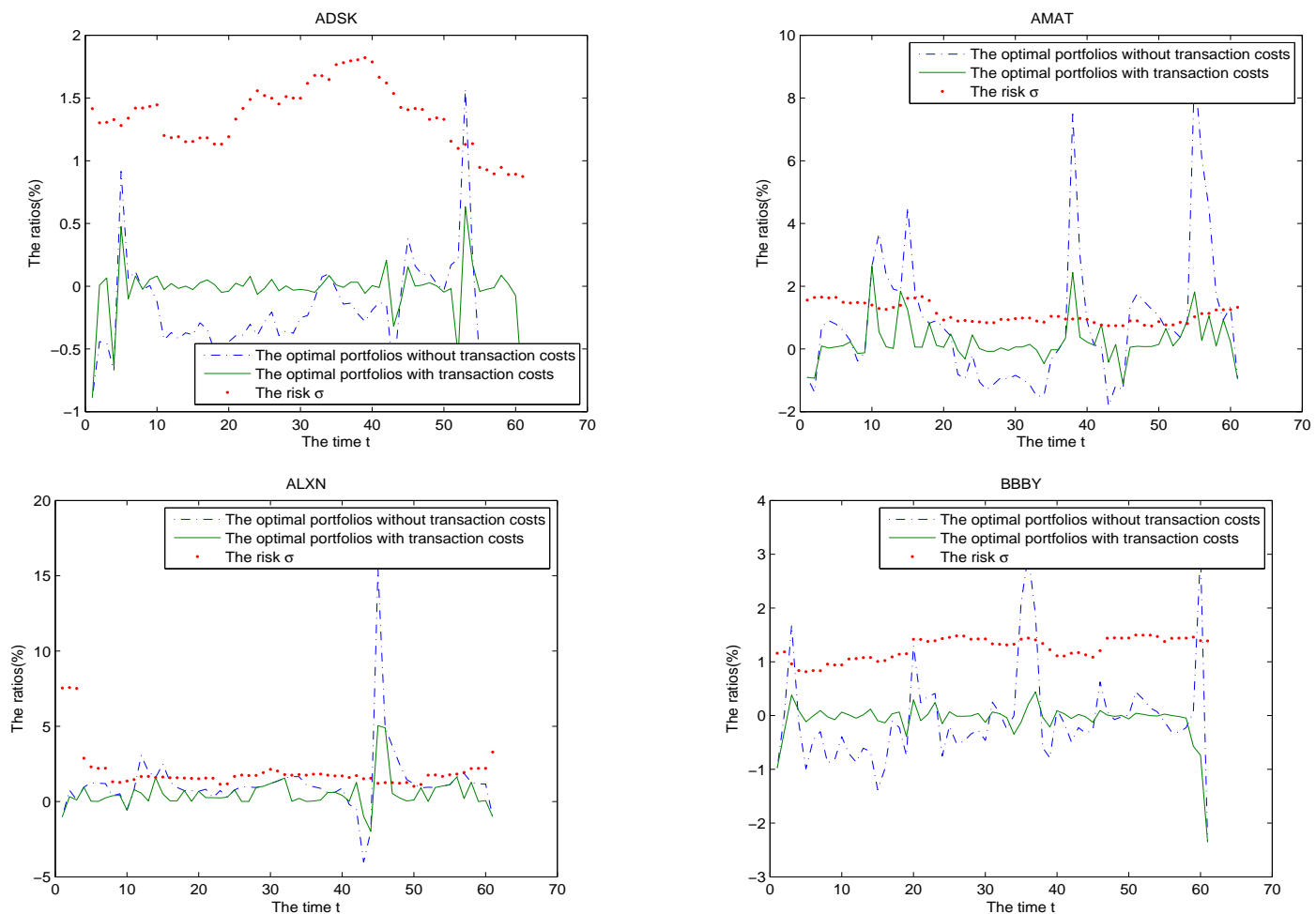

Figure 1. The optimal portfolios without transaction costs versus the optimal portfolios with transaction costs

The similarity: Both the optimal portfolios without transaction costs and the optimal portfolios with transaction costs are sensitive to the risks of the risky asset. When these risks increase, regardless of whether we consider transaction costs, the CPT-investor decreases the amount of investment in the risky asset and increases the amount of investment in the risk-free asset. The CPT-investor hopes to reduce the risk of his portfolios through this change in the investment strategy. In contrast, when the risks of the risky asset decrease, the CPT-investor increases the amount of investment in the risky asset and reduces their investment in risk-free assets to obtain more excess returns of risky assets.

The difference: The optimal portfolios with trade costs are less sensitive to the risks of the risky asset than the optimal portfolios without trade costs. When the risks of the risk asset decrease, considering trade costs, the CPT-investor additionally invests less money in the risky asset than the CPT-investor who does not consider trade costs. In contrast, when the risks of the risky asset increase, considering transaction costs, the CPT-investor additionally invests less money in the risk-free asset than the CPT-investor who does not consider transaction costs. When transaction costs exist, adjusting portfolios too frequently will increase the total costs and decrease the return at the terminal time. Therefore, the CPTinvestor will decrease the frequency of transactions and the adjusted amount of investment. Therefore, the CPT-investor who considers transaction costs is less sensitive to the risks of the risky asset than the CPT-investor who does not consider transaction costs. This conclusion is consistent with the classical result that increasing transaction costs decrease the liquidity of assets.

\section{Conclusions}

The time-changing transaction costs play a significant role in the multi-period strategy under CPT framework. Because 
the amount of the investment in the different time, the transaction costs are different in the different time. Due to the different transaction costs, the benchmark is time-changing. Furtherly,we have to deal with the problem that the timing of the investment strategy. We propose CPT recursive solution with transaction costs under the multiple periods. Besides, through the numerical analysis, we find that the optimal strategy with transaction costs is less sensitive to risks than the optimal strategy without transaction costs regardless of different levels of risks and risk volatilities. The result is consistent with the classical conclusion that transaction costs reduce the liquidity of assets.

\section{Acknowledgements}

The article is supported by Humanities and social science project of Ministry of Education (No.17YJC790020).

\section{Reference}

Bernard, C., \& Ghossoub, M. (2009). Static portfolio choice under cumulative prospect theory. MPRA Paper 16502, Munich personal RePEc Archive. https://doi.org/10.1007/s11579-009-0021-2

Carassus, L. R., \& ásonyi (2013). On Optimal Investment for a Behavioural Investor in Multiperiod Incomplete Market Models. Math Financ, arXiv preprint arXiv:1608.08490. https://doi.org/10.1111/mafi.12018

Chateauneuf, A., Dana, R. A., \& Tallon, J. (2000). Optimal risk-sharing rules and equilibria with Choquet-expectedutility. J Math Econ, 34(2), 191-214.

Choquet, G. (1953). Theory of capacities. Ann I Fourier, 5, 131-295.

Deng, L., \& Pirvu, T. A. (2015). Multi-period investment strategies under Cumulative Prospect Theory. Working paper, arXiv preprint arXiv:1608.08490.

Davis, M., \& Norman, A. (1990). Portfolio selection with transaction costs. Mathematics of Operations Research, 15(4), 676-713. https://doi.org/10.1287/moor.15.4.676

He, X., \& Zhou, X. (2011a). Portfolio choice under cumulative prospect theory: An analytical treatment. Manage Sci, 57(2), 315-331. https://doi.org/10.2139/ssrn.1479580

He, X., \& Zhou, X. (2011b). Portfolio choice via quantiles. Math Financ, 21(2), 203-231.

Jin, H., \& Zhou, X. (2008). Behavioral portfolio selection in continuous time. Math Financ, 18(3), 385-426. https://doi.org/10.1111/j.1467-9965.2008.00339.x

Kabanov, Y., \& Safarian, M. (2010). Markets with transaction Costs. Springer-Verlag Berlin Heidelberg. https://doi.org/10.1007/978-3-540-68121-2

Magill, M., \& Constantinides, G. (1976). Portfolio selection with transaction costs. Journal of Economic Theory, 13, 245-263. https://doi.org/10.1016/0022-0531(76)90018-1

Neummann, J., \& Morgenstern, O. (1944). Theory of games and economic behavior. Princeton University Press, Princeton, NJ.

Pirvu, T. A., \& Schulze, K. (2012). Multi-stock portfolio optimization under prospect theory. Math Financ Econ, 6, 337-362. https://doi.org/10.1007/s11579-012-0079-0

Shreve, S., \& Soner, M. (1994) Optimal investment and consumption with transaction costs. Annals of Applied Probability, $4(3), 609-692$.

Shi, Y., Cui, X., \& Li, D. (2014). Discrete-time behavioral portfolio selection under prospect theory. Working paper, available at SSRN 2457762. https://doi.org/10.2139/ssrn.2457762

Tversky, A., \& Kahneman, D. (1992a). Prospect theory: An analysis of decision under rick. Econometrica, 47, 262-291. https://doi.org/10.1007/BF00122574

Tversky, A., \& Kahneman, D. (1992b). Advances in prospect theory: Cumulative representation of uncertainty. J Risk Uncertainty, 5, 297-323.

Zou, B., \& Zagst, R. (2017). Optimal investment with transaction costs under cumulative prospect theory in discrete time. Math Financ Econ. First online. https://doi.org/10.1007/s11579-017-0186-z. 


\section{Appendix}

Appendix A: Proof of Proposition 0.1

Proof. We denote the benchmark at time $T$ by $\bar{W}_{T-k}^{T}$ when the initial time is $T-k$. Now, by mathematical induction, we prove

$$
\bar{W}_{T-k}^{T}=z_{T-k}^{T} \theta_{T-k}+z_{T-k+1}^{T} \theta_{T-k+1}+\ldots+z_{T-1}^{T} \theta_{T-1} .
$$

When $k=1$, then (4) indicates

$$
\bar{W}_{T-1}^{T}=W_{T}-B_{T-1}^{T}=z_{T-1}^{T} \theta_{T-1} .
$$

That is, (20) holds.

Moreover, when $k=2$, with equation (8), one can show that

$$
\bar{W}_{T-2}^{T}=W_{T}-B_{T-2}^{T}=z_{T-2}^{T} \theta_{T-2}+z_{T-1}^{T} \theta_{T-1} .
$$

We suppose when $k=N$, (20) holds. That is,

$$
\bar{W}_{T-N}^{T}=W_{T}-B_{T-N}^{T}=z_{T-N}^{T} \theta_{T-N}+z_{T-N+1}^{T} \theta_{T-N+1}+\ldots+z_{T-1}^{T} \theta_{T-1} .
$$

Now, we only need to prove that equation (20) holds when $k=N+1$.

With

$$
\bar{W}_{T-N}^{T}=W_{T}-B_{T-N}^{T}
$$

and

$$
\bar{W}_{T-N-1}^{T}=W_{T}-B_{T-N-1}^{T},
$$

one can easily obtain

$$
\begin{aligned}
\bar{W}_{T-N-1}^{T} & =W_{T}-B_{T-N}^{T}+B_{T-N}^{T}-B_{T-N-1}^{T} \\
& =\bar{W}_{T-N}^{T}+B_{T-N}^{T}-B_{T-N-1}^{T} \\
& =z_{T-N}^{T} \theta_{T-N}+z_{T-N+1}^{T} \theta_{T-N+1}+\ldots+z_{T-1}^{T} \theta_{T-1}+B_{T-N}^{T}-B_{T-N-1}^{T} .
\end{aligned}
$$

Hence, we only need to prove

$$
B_{T-N}^{T}-B_{T-N-1}^{T}=z_{T-N-1}^{T} \theta_{T-N-1} .
$$

We have

$$
\begin{aligned}
& B_{T-N}^{T}-B_{T-N-1}^{T} \\
= & \left(1+r_{T-N}\right)\left(1+r_{T-N+1}\right) \ldots\left(1+r_{T-1}\right) x_{T-N} \\
+ & \left(1+R_{T-N}\right)\left(1+R_{T-N+1}\right) \ldots\left(1+R_{T-1}\right)\left(y_{T-N}-\lambda y_{T-N}^{+}\right) \\
- & \left(1+r_{T-N-1}\right)\left(1+r_{T-N}\right) \ldots\left(1+r_{T-1}\right) x_{T-N-1} \\
- & \left(1+R_{T-N-1}\right)\left(1+R_{T-N}\right) \ldots\left(1+R_{T-1}\right)\left(y_{T-N-1}-\lambda y_{T-N-1}^{+}\right) .
\end{aligned}
$$

Applying

$$
x_{T-N}=\left(1+r_{T-N-1}\right)\left(x_{T-N-1}-\theta_{T-N-1}\right)
$$

and

$$
y_{T-N}=\left(1+R_{T-N-1}\right)\left(y_{T-N-1}+\theta_{T-N-1}\right)
$$


to (27), we can obtain that

$$
\begin{aligned}
& B_{T-N}^{T}-B_{T-N-1}^{T} \\
= & -\left(1+r_{T-N-1}\right)\left(1+r_{T-N}\right) \ldots\left(1+r_{T-1}\right) \theta_{T-N-1} \\
+ & (1-\lambda)\left(1+R_{T-N-1}\right)\left(1+R_{T-N}\right) \ldots\left(1+R_{T-1}\right) \theta_{T-N-1} \\
= & z_{T-N-1}^{T} \theta_{T-N-1} .
\end{aligned}
$$

Therefore, we show that equation (20) holds when $k=N+1$.

Appendix B: Proof of Proposition 0.2

Proof. Let $\theta_{T-1}=W_{T-1} s$.

Case 1: $\theta_{T-1} \geq 0$

When $W_{T-1} \geq 0$, we directly employ (14) in order to obtain

$$
\begin{aligned}
& U\left(\bar{W}_{T-1}^{T}\left(\theta_{T-1}\right)\right) \\
= & \theta_{T-1}^{\alpha}\left(\int_{0}^{+\infty} T^{+}\left(1-F_{z_{T-1}^{T}}(x)\right) d u^{+}(x)-\int_{0}^{+\infty} T^{-}\left(F_{z_{T-1}^{T}}(-x)\right) d u^{-}(x)\right) .
\end{aligned}
$$

Therefore,

$$
\begin{aligned}
& U\left(\bar{W}_{T-1}^{T}\left(\theta_{T-1}\right)\right) \\
= & W_{T-1}^{\alpha} s^{\alpha}\left(\int_{0}^{+\infty} T^{+}\left(1-F_{z_{T-1}^{T}}(x)\right) d u^{+}(x)-\int_{0}^{+\infty} T^{-}\left(F_{z_{T-1}^{T}}(-x)\right) d u^{-}(x)\right) . \\
= & W_{T-1}^{\alpha} s^{\alpha} h(T-1)
\end{aligned}
$$

Hence, when $W_{T-1} \geq 0$, we have that

$$
U\left(\bar{W}_{T-1}^{T}\left(v_{T-1}\right)\right)=W_{T-1}^{\alpha} s^{\alpha} h(T-1) I_{s \in[0,+\infty)}=W_{T-1}^{\alpha} g_{T-1}(s),
$$

where $g_{T-1}(s)=s^{\alpha} h(T-1) I_{s \in[0,+\infty)}$.

When $W_{T-1}<0$, one easily obtains $\theta_{T-1}=\left(-W_{T-1}\right)(-s)$ and

$$
\begin{aligned}
& U\left(\bar{W}_{T-1}^{T}\left(\theta_{T-1}\right)\right) \\
= & \left(-W_{T-1}\right)^{\alpha}(-s)^{\alpha}\left(\int_{0}^{+\infty} T^{+}\left(1-F_{z_{T-1}^{T}}(x)\right) d u^{+}(x)-\int_{0}^{+\infty} T^{-}\left(F_{z_{T-1}^{T}}(-x)\right) d u^{-}(x)\right) \\
= & \left(-W_{T-1}\right)^{\alpha}(-s)^{\alpha} h(T-1)
\end{aligned}
$$

Therefore, when $W_{T-1}<0$, we have that

$$
\begin{aligned}
U\left(\bar{W}_{T-1}^{T}\left(\theta_{T-1}\right)\right) & =\left(-W_{T-1}\right)^{\alpha}(-s)^{\alpha} h(T-1) I_{s \in(-\infty, 0]} \\
& =\left(-W_{T-1}\right)^{\alpha} l_{T-1}(s),
\end{aligned}
$$

where $l_{T-1}(s)=(-s)^{\alpha} h(T-1) I_{s \in(-\infty, 0]}$.

Case 2: $\theta_{T-1}<0$

When $W_{T-1} \geq 0$, with (14), we obtain $W_{T-1}=\left(-\theta_{T-1}\right)\left(-z_{T-1}^{T}\right)$ and

$$
\begin{aligned}
& U\left(\bar{W}_{T-1}^{T}\left(\theta_{T-1}\right)\right) \\
= & \left(-\theta_{T-1}\right)^{\alpha}\left(\int_{0}^{+\infty} T^{+}\left(1-F_{-z_{T-1}^{T}}(x)\right) d u^{+}(x)-\int_{0}^{+\infty} T^{-}\left(F_{z_{T-1}^{T}}(x)\right) d u^{-}(x)\right) .
\end{aligned}
$$


Therefore,

$$
\begin{aligned}
& U\left(\bar{W}_{T-1}^{T}\left(\theta_{T-1}\right)\right) \\
= & W_{T-1}^{\alpha}(-s)^{\alpha}\left(\int_{0}^{+\infty} T^{+}\left(1-F_{-z_{T-1}^{T}}(x)\right) d u^{+}(x)-\int_{0}^{+\infty} T^{-}\left(F_{z_{T-1}^{T}}(x)\right) d u^{-}(x)\right) . \\
= & W_{T-1}^{\alpha}(-s)^{\alpha} k(T-1)
\end{aligned}
$$

Hence, when $W_{T-1} \geq 0$, we have that

$$
U\left(\bar{W}_{T-1}^{T}\left(v_{T-1}\right)\right)=W_{T-1}^{\alpha}(-s)^{\alpha} k(T-1) I_{s \in(-\infty, 0]}=W_{T-1}^{\alpha} g_{T-1}(s),
$$

where $g_{T-1}(s)=(-s)^{\alpha} k(T-1) I_{s \in(-\infty, 0]}$.

When $W_{T-1}<0$, one easily obtains $\theta_{T-1}=\left(-W_{T-1}\right)(-s)$ and

$$
\begin{aligned}
& U\left(\bar{W}_{T-1}^{T}\left(\theta_{T-1}\right)\right) \\
= & \left(-W_{T-1}\right)^{\alpha} s^{\alpha}\left(\int_{0}^{+\infty} T^{+}\left(1-F_{-z_{T-1}^{T}}(x)\right) d u^{+}(x)-\int_{0}^{+\infty} T^{-}\left(F_{z_{T-1}^{T}}(x)\right) d u^{-}(x)\right) \\
= & \left(-W_{T-1}\right)^{\alpha} s^{\alpha} k(T-1)
\end{aligned}
$$

Therefore, when $W_{T-1}<0$, we have that

$$
\begin{aligned}
U\left(\bar{W}_{T-1}^{T}\left(\theta_{T-1}\right)\right) & =\left(-W_{T-1}\right)^{\alpha} s^{\alpha} k(T-1) I_{s \in(0,+\infty]} \\
& =\left(-W_{T-1}\right)^{\alpha} l_{T-1}(s),
\end{aligned}
$$

where $l_{T-1}(s)=s^{\alpha} k(T-1) I_{s \in[0,+\infty)}$.

Appendix C: Proof of Proposition 0.4

Proof. We prove this by mathematical induction. Proposition 0.2 displays that the conclusion of Proposition 0.4 holds at time $\mathrm{t}-1$. We suppose that the conclusion holds at the time $t+1$. In other words,

$$
\max _{\theta_{t+1}, \theta_{t+2}, \ldots \theta_{T-1}} U\left(\underline{W}_{t+1}^{T}\right)=A_{t+1} W_{t+1}^{\alpha} I_{W_{t+1} \geq 0}-B_{t+1}\left(-W_{t+1}\right)^{\alpha} I_{W_{t+1}<0}
$$

Since

$$
\underline{W}_{t}^{T}=\theta_{T-1} z_{T-1}^{T} \quad \text { given that the initial time is } t
$$

iterated conditioning yields

$$
\begin{aligned}
& \max _{\theta_{t}, \theta_{t+1}, \ldots \theta_{T-1}} U\left(\underline{W}_{t}^{T}\right) \\
= & \max _{\theta_{t}} E_{t}\left[\max _{\theta_{t+1}, \theta_{t+2}, \ldots \theta_{T-1}} U\left(\underline{W}_{t+1}^{T}\right)\right] \\
= & \max _{\theta_{t}} E\left[A_{t+1} W_{t+1}^{\alpha} I_{W_{t+1} \geq 0}-B_{t+1}\left(-W_{t+1}\right)^{\alpha} I_{W_{t+1}<0} \mid \mathcal{F}_{t}\right] .
\end{aligned}
$$

Let

$$
y_{t}+\theta_{t}=s W_{t}
$$

Since

$$
W_{t}=x_{t}+y_{t}
$$

we have

$$
x_{t}-\theta_{t}=W_{t}-y_{t}-\theta_{t}=W_{t}-\left(y_{t}+\theta_{t}\right)=(1-s) W_{t} .
$$


Case 1: $\theta_{t} \geq 0$

From equation (1), one can obtain that

$$
\begin{aligned}
W_{t+1} & =\left(1+r_{t}\right)\left(x_{t}-\theta_{t}\right)+\left(1+R_{t}\right)\left(y_{t}+\theta_{t}\right) \\
& -\lambda\left[\left(1+R_{t}\right)\left(y_{t}+\theta_{t}\right)^{+}+\left(1+r_{t}\right) \theta_{t}^{-}\right] \\
& =\left(1+r_{t}\right)(1-s) W_{t}+\left(1+R_{t}\right) s W_{t} \\
& -\lambda\left(1+R_{t}\right)\left(s W_{t}\right)^{+}
\end{aligned}
$$

When $W_{t} \geq 0$, due to a lack of short-selling, i.e., $y_{t}+\theta_{t} \geq 0$, it is easy to show that

$$
\begin{aligned}
& W_{t+1}=\left[\left(1+r_{t}\right)(1-s)+\left(1+R_{t}\right) s-\lambda\left(1+R_{t}\right) s^{+}\right] W_{t} . \\
& \max _{\theta_{t}, \ldots, \theta_{T-1}} U\left(\underline{W}_{t}^{T}\right) \\
= & W_{t}^{\alpha} \max _{s \in(-\infty,+\infty)} E\left[\left(\left(1+r_{t}\right)(1-s)+\left(1+R_{t}\right) s-\lambda\left(1+R_{t}\right) s^{+}\right)^{\alpha} .\right. \\
& A_{t+1} I_{\left(1+r_{t}\right)(1-s)+\left(1+R_{t}\right) s-\lambda\left(1+R_{t}\right) s^{+} \geq 0} \\
- & {\left[-\left(1+r_{t}\right)(1-s)-\left(1+R_{t}\right) s+\lambda\left(1+R_{t}\right) s^{+}\right]^{\alpha} . } \\
= & \left.B_{t+1} I_{\left(1+r_{t}\right)(1-s)+\left(1+R_{t}\right) s-\lambda\left(1+R_{t}\right) s^{+}<0} \mid \mathcal{F}_{t}\right] \\
= & W_{t}^{\alpha} \max _{s \in(-\infty,+\infty)}^{\alpha} A_{t} .
\end{aligned}
$$

When $W_{t}<0$, similarly, we have

$$
x_{t}-\theta_{t}=(s-1)\left(-W_{t}\right) \text {. }
$$

From equation (1), one can obtain that

$$
\begin{aligned}
W_{t+1} & =\left(1+r_{t}\right)(s-1)\left(-W_{t}\right)+\left(1+R_{t}\right)(-s)\left(-W_{t}\right) \\
& -\lambda\left(1+R_{t}\right)\left(s W_{t}\right)^{+} \\
& =\left[\left(1+r_{t}\right)(s-1)+\left(1+R_{t}\right)(-s)\right. \\
& \left.-\lambda\left(1+R_{t}\right)(-s)^{+}\right]\left(-W_{t}\right)
\end{aligned}
$$

we confirm that

$$
\begin{aligned}
& \max _{\theta_{t}, \ldots, \theta_{T-1}} U\left(\underline{W}_{t}^{T}\right) \\
= & \left(-W_{t}\right)^{\alpha} \max _{s \in(-\infty,+\infty)} E\left[\left(\left(1+r_{t}\right)(s-1)+\left(1+R_{t}\right)(-s)-\lambda\left(1+R_{t}\right)(-s)^{+}\right)^{\alpha} .\right. \\
& A_{t+1} I_{\left(1+r_{t}\right)(s-1)+\left(1+R_{t}\right)(-s)-\lambda\left(1+R_{t}\right)(-s)^{+}>0} \\
- & \left(-\left(1+r_{t}\right)(s-1)-\left(1+R_{t}\right)(-s)+\lambda\left(1+R_{t}\right)(-s)^{+}\right)^{\alpha} . \\
& \left.B_{t+1} I_{\left(1+r_{t}\right)(s-1)+\left(1+R_{t}\right)(-s)-\lambda\left(1+R_{t}\right)(-s)^{+} \leq 0} \mid \mathcal{F}_{t}\right] \\
= & \left(-W_{t}\right)^{\alpha} \max _{s \in(-\infty,+\infty)} l_{t}(s) \\
= & -\left(-W_{t}\right)^{\alpha} B_{t} .
\end{aligned}
$$

Case 2: $\theta_{t}<0$

From equation(1), we have

$$
\begin{aligned}
W_{t+1} & =\left(1+r_{t}\right)\left(x_{t}-\theta_{t}\right)+\left(1+R_{t}\right)\left(y_{t}+\theta_{t}\right) \\
& -\lambda\left[\left(1+R_{t}\right)\left(y_{t}+\theta_{t}\right)^{+}-\left(1+r_{t}\right) \theta_{t}\right]
\end{aligned}
$$


Let

$$
y_{t}+\theta_{t}=s W_{t}
$$

and

$$
\theta_{t}=k W_{t}
$$

We have

$$
y_{t}+k W_{t}=s W_{t} .
$$

Therefore,

$$
k=s-\frac{y_{t}}{W_{t}} .
$$

With Taylor expansion of $\frac{y_{t}}{W_{t}}$ at $W_{t}=1$, one can obtain that

$$
k \approx s-y \text {. }
$$

we have

$$
x_{t}-\theta_{t}=W_{t}-y_{t}-\theta_{t}=W_{t}-\left(y_{t}+\theta_{t}\right)=(1-s) W_{t} .
$$

From equation (50), one can obtain that

$$
\begin{aligned}
W_{t+1} & =\left(1+r_{t}\right)\left(x_{t}-\theta_{t}\right)+\left(1+R_{t}\right)\left(y_{t}+\theta_{t}\right) \\
& -\lambda\left[\left(1+R_{t}\right)\left(y_{t}+\theta_{t}\right)^{+}+\left(1+r_{t}\right) \theta_{t}^{-}\right] \\
& =\left(1+r_{t}\right)(1-s) W_{t}+\left(1+R_{t}\right) s W_{t} \\
& -\lambda\left(1+R_{t}\right)\left(s W_{t}\right)^{+}+\lambda\left(1+r_{t}\right)\left(s-y_{t}\right) W_{t}
\end{aligned}
$$

When $W_{t} \geq 0$, due to a lack of short-selling, i.e., $y_{t}+\theta_{t} \geq 0$, it is easy to show that

$$
\begin{aligned}
W_{t+1}= & {\left[\left(1+r_{t}\right)(1-s)+\left(1+R_{t}\right) s-\lambda\left(1+R_{t}\right) s^{+}+\lambda\left(1+r_{t}\right)\left(s-y_{t}\right)\right] W_{t} . } \\
& \max _{\theta_{t}, \ldots, \theta_{T-1}} U\left(\underline{W}_{t}^{T}\right) \\
= & W_{t}^{\alpha} \max _{s \in(-\infty,+\infty)} E\left[\left(\left(1+r_{t}\right)(1-s)+\left(1+R_{t}\right) s-\lambda\left(1+R_{t}\right) s^{+}\right.\right. \\
+ & \left.\lambda\left(1+r_{t}\right)\left(s-y_{t}\right)\right)^{\alpha} A_{t+1} I_{\left(1+r_{t}\right)(1-s)+\left(1+R_{t}\right) s-\lambda\left(1+R_{t}\right) s^{+}+\lambda\left(1+r_{t}\right)\left(s-y_{t}\right) \geq 0} \\
& -\left(-\left(1+r_{t}\right)(1-s)-\left(1+R_{t}\right) s+\lambda\left(1+R_{t}\right) s^{+}\right. \\
& \left.\left.-\lambda\left(1+r_{t}\right)\left(s-y_{t}\right)\right)^{\alpha} B_{t+1} I_{\left(1+r_{t}\right)(1-s)+\left(1+R_{t}\right) s-\lambda\left(1+R_{t}\right) s^{+}+\lambda\left(1+r_{t}\right)\left(s-y_{t}\right)<0} \mid \mathcal{F}_{t}\right] \\
= & W_{t}^{\alpha} \max _{s \in(-\infty,+\infty)} g_{t}(s) \\
= & W_{t}^{\alpha} A_{t} .
\end{aligned}
$$

When $W_{t}<0$, similarly,

we have

$$
x_{t}-\theta_{t}=(s-1)\left(-W_{t}\right)
$$

From equation (1), one can obtain that

$$
\begin{aligned}
W_{t+1} & =\left(1+r_{t}\right)(s-1)\left(-W_{t}\right)+\left(1+R_{t}\right)(-s)\left(-W_{t}\right) \\
& -\lambda\left(1+R_{t}\right)\left(s W_{t}\right)^{+}+\lambda\left(1+r_{t}\right)\left(y_{t}-s\right)\left(-W_{t}\right) \\
& =\left[\left(1+r_{t}\right)(s-1)+\left(1+R_{t}\right)(-s)\right. \\
& \left.-\lambda\left(1+R_{t}\right)(-s)^{+}+\lambda\left(1+r_{t}\right)\left(y_{t}-s\right)\right]\left(-W_{t}\right)
\end{aligned}
$$


we confirm that

$$
\begin{aligned}
& \max _{\theta_{t}, \ldots, \theta_{T-1}} U\left(\underline{W}_{t}^{T}\right) \\
= & \left(-W_{t}\right)^{\alpha} \max _{s \in(-\infty,+\infty)} E\left[\left(\left(1+r_{t}\right)(s-1)+\left(1+R_{t}\right)(-s)-\lambda\left(1+R_{t}\right)(-s)^{+}\right.\right. \\
+ & \left.\lambda\left(1+r_{t}\right)\left(y_{t}-s\right)\right)^{\alpha} A_{T-1} I_{\left(1+r_{t}\right)(s-1)+\left(1+R_{t}\right)(-s)-\lambda\left(1+R_{t}\right)(-s)^{+}+\lambda\left(1+r_{t}\right)\left(y_{t}-s\right)>0} \\
- & \left(-\left(1+r_{t}\right)(s-1)-\left(1+R_{t}\right)(-s)+\lambda\left(1+R_{t}\right)(-s)^{+}\right. \\
- & \left.\left.\lambda\left(1+r_{t}\right)\left(y_{t}-s\right)\right)^{\alpha} B_{t+1} I_{\left(1+r_{t}\right)(s-1)+\left(1+R_{t}\right)(-s)-\lambda\left(1+R_{t}\right)(-s)^{+}+\lambda\left(1+r_{t}\right)\left(y_{t}-s\right) \leq 0} \mid \mathcal{F}_{t}\right] \\
= & \left(-W_{t}\right)^{\alpha} \max _{s \in(-\infty,+\infty)} l_{t}(s) \\
= & -\left(-W_{t}\right)^{\alpha} B_{t} .
\end{aligned}
$$

Therefore, Proposition 0.4 holds.

\section{Copyrights}

Copyright for this article is retained by the author(s), with first publication rights granted to the journal. This is an open-access article distributed under the terms and conditions of the Creative Commons Attribution license which permits unrestricted use, distribution, and reproduction in any medium, provided the original work is properly cited. 